\title{
Significados, causas y efectos de la violencia social entre la juventud en Monterrey, Nuevo León, México ${ }^{1}$
}

\author{
Meanings, Causes and Effects of Social \\ Violence Among Youth in Monterrey, Nuevo \\ Leon, Mexico
}

\section{Significados, causas e efeitos da violência entre a juventude em Monterrey, Nuevo León, México}

\author{
Jesús Rubio Campos ${ }^{2}$ \\ El Colegio de la Frontera Norte A.C., México \\ rubio@colef.mx \\ Mónica Chávez Elorza \\ Universidad Autónoma de Zacatecas, México \\ monica.chavez@estudiosdeldesarrollo.net
}

\begin{abstract}
Héctor Rodríguez Ramírez ${ }^{4}$
Tecnológico de Monterrey, México

hrr@itesm.mx
\end{abstract}

Recibido: 14/04/16

Aprobado: 28/10/2016

\footnotetext{
1 El presente trabajo se deriva de un proyecto de investigación realizado como parte de las actividades del Legado de Seguridad y Estado Constitucional de Derecho del Tecnológico de Monterrey, que fue financiado con fondos federales del Subsidio para la Seguridad Pública de los Municipios (SUBSEMUN) de México.

2 Doctor en Política Pública.

3 Doctora en Política Pública.

4 Doctor en Ciencias Sociales.
} 


\title{
Resumen
}

El objetivo de la presente investigación es analizar los diferentes significados, causas y efectos de la violencia social desde el punto de vista de la juventud, que sirvan para establecer estrategias de prevención. Para tal fin, se utilizó un estudio multicaso, a partir de trabajo de campo en tres barrios considerados como zonas de atención prioritaria en la ciudad de Monterrey. Para este análisis, se utilizó una metodología cualitativa, que en la primera etapa consistió en observación planificada y entrevistas con vecinos y personal de centros comunitarios. En la segunda etapa, se desarrollaron talleres participativos con jóvenes. Los resultados de la investigación mostraron que los jóvenes reconocen diversos tipos de violencia, y tienen claridad sobre sus causas -sean biológicas, familiares, personales, sociales o institucionales-; también son conscientes de los efectos de la violencia -de índole psicológica individual, la reafirmación de actitudes violentas, incremento de las adicciones-, así como la desintegración de la familia, su círculo de amigos y su comunidad.

Palabras clave: violencia; prevención del crimen; juventud; participación comunitaria; Monterrey.

\begin{abstract}
The aim of this research is to analyze the different meanings, causes and effects of social violence from the point of view of youth, which serve to implement prevention strategies. To this end, the cases of three colonies considered priority attention areas in the city of Monterrey were analyzed. A qualitative methodology was developed in a first stage, consisted of planned observation and interviews with neighbors and community centers staff; in a second stage, participatory workshops with youth were developed. The results of the research showed that young people recognize different types of violence, they are clear about their causes, -whether biological, familiar, personal, social or institutional-and their effects, psychological at the individual level, reaffirmation of violent attitudes and an increase in addictions; others at social level such as families, circles of friends and communities disintegration.
\end{abstract}

Keywords: Violence; Crime Prevention; Youth; Citizen Participation; Monterrey.

\section{Resumo}

O objetivo da presente pesquisa e analisar os diferentes significados, causas e efeitos da violência social desde o ponto de vista da juventude, para estabelecer estratégias de prevenção. Nesse sentido, foram analisados os casos das colônias que representam zonas de atenção prioritária em Monterrey, México. Uma metodologia qualitativa foi implementada numa primeira etapa com observações planificadas e entrevistas com vizinhos e pessoal de centros comunitários. Na segunda etapa, foram implementadas oficinas participativas com jovens. Os resultados da pesquisa revelaram que os jovens reconhecem vários tipos de violência, identificando as causas- biológicas, familiares, pessoais, sociais ou institucionais- e os efeitos no nível psicológico-individual, além da reafirmação das atitudes violentas, um incremento nas adições, a desintegração das relações familiares, de amizade e comunitária.

Palavras chaves: violência; prevenção ao crime; juventude; participação da comunidade; Monterrey.

Este trabajo está bajo la licencia Creative Commons Attribution 3.0

¿Cómo citar este artículo? / How to quote this article?

Rubio-Campos, J., Chávez-Elorza, M. y Rodríguez-Ramírez, H. (2017). Significados, causas y efectos de la violencia social entre la juventud en Monterrey, Nuevo León, México. Sociedad y economía, (32), 85-106. 


\section{Introducción}

Desde 2008, el fenómeno de la violencia y la inseguridad en México se ha posicionado como tema prioritario tanto en la agenda pública como en la percepción de la ciudadanía. De acuerdo con datos del Sistema Nacional de Seguridad Pública (SNSP), durante el período 2001-2011, la incidencia delictiva del fuero federal aumentó en $83 \%$, pasando de 78.309 a 142.971 casos; así mismo hubo un aumento en $538 \%$ en el presupuesto destinado a la Secretaría de Seguridad Pública Federal (63 miles de millones de pesos mexicanos -mmdp- a 40,5 mmdp) (Comisión Intersecretarial para la Prevención Social de la Violencia y la Delincuencia -CIPSVD-, 2013, p. 17). Dentro de este fenómeno, la violencia entre las juventudes se ha convertido en un problema público grave en México.

Los jóvenes sobresalen tanto por ser los perpetradores como por ser las víctimas de delitos. Según cifras del Instituto Nacional de Estadística, Geografía e Informática (INEGI) de México, durante el período 2010-2015 se registraron en el país 135.677 defunciones por homicidio, de las cuales $88 \%$ fueron hombres, $11 \%$ mujeres y el restante $1 \%$ no fue identificado. Del total de las defunciones, $38,4 \%$ corresponde a personas entre 10 y 29 años. En el caso particular de Nuevo León se registraron 6.846 defunciones por homicidio en el mismo período 2010-2015, de las cuales $86,6 \%$ se presentaron en hombres y el resto en mujeres. Del total de defunciones, 33,0\% se presentó entre personas de 10 a 29 años (INEGI, 2016a).

Por su parte, los registros sobre delincuentes sentenciados en el fuero común en 2008 muestran que, a escala nacional, de los 134.221 sentenciados, $91,0 \%$ se trató de hombres y 9,0\% de mujeres; asimismo, 65,9\% del total de ellos tenía entre 16 y 29 años edad. En el caso de Nuevo León, de los 2.546 delincuentes sentenciados en el fuero común, $94,6 \%$ se trató de hombres; y del total, 57,8\% de ellos tenía entre 18 y 29 años de edad (INEGI, 2016b). En conjunto, estas cifras ponen de manifiesto la importancia de comprender los significados de la violencia para diseñar e implementar estrategias que busquen disminuir estas tendencias.

En la presente investigación se analiza el fenómeno de la violencia en la juventud que reside en tres colonias de la ciudad de Monterrey: Fomerrey 45, La Alianza y La Independencia. La selección de estas tres colonias se basó en que fueron catalogadas por la Secretaría de Desarrollo Social (Sedesol, federal), como zonas de Atención Prioritaria Urbana (ZAPU). Este programa atiende integralmente los rezagos vinculados con la infraestructura básica comunitaria y la carencia de servicios básicos en las viviendas ubicadas en los municipios de muy alta y alta marginación ${ }^{5}$.

Este artículo está organizado en cuatro secciones. En una primera parte, se presenta el marco teórico sobre la violencia, sus definiciones, clasificaciones, causas y manifestaciones. En la segunda, se presenta un breve antecedente y marco contextual de los territorios analizados y la estrategia metodológica utilizada. La tercera parte consta de los resultados del trabajo de campo; en primera instancia se presentan las principales problemáticas percibidas por la juventud en sus colonias, seguido de los significados que dan a la violencia, presentando

5 El programa ZAPU, junto con los programas de Desarrollo Local y Microrregiones se fusionaron para conformar el Programa para el Desarrollo de Zonas Prioritarias (PDZP). Para mayor detalle al respecto consultar el sitio de la Secretaría de Desarrollo Social: http://www.sedesol.gob.mx/ es/SEDESOL/Programa_para_el_Desarrollo_de_Zonas_Prioritarias_PDZP 
y analizando sus diversas dimensiones de acuerdo con el marco teórico planteado; enseguida, se presentan los factores promotores de la violencia y sus efectos, siempre desde la visión de las y los jóvenes; se finaliza esta sección con la presentación de algunos proyectos que a consideración de la juventud participante en los talleres pueden atenuar la violencia en su entorno. La cuarta parte, y a modo de conclusión, presenta una reflexión sobre los hallazgos del trabajo de campo, así como la manera en que los marcos teóricos existentes contribuyen a la comprensión de este fenómeno y las limitaciones que presentan.

\section{Marco teórico}

Según la Organización Panamericana de la Salud, la violencia es una de las principales causas de muerte en el mundo y origina problemas no solo a las víctimas directas de la misma, sino a su círculo familiar, su entorno laboral y en general a la comunidad donde se presenta (OPS, 2002, p. 1). La Organización Mundial de la Salud (OMS, 1996, citado en OPS, 2002) define a la violencia como: "El uso deliberado de la fuerza física o el poder, ya sea en grado de amenaza o efectivo, contra uno mismo, otra persona o un grupo o comunidad, que cause o tenga muchas probabilidades de causar lesiones, muerte, daños psicológicos, trastornos del desarrollo o privaciones" (p. 5). Esta organización divide la violencia en tres grandes categorías según el autor del acto violento: violencia dirigida contra uno mismo, violencia interpersonal y violencia colectiva. Así mismo, señala que la naturaleza de la violencia puede ser de índole física, sexual, psicológica y privativa o de desatención.

De manera similar, Buvinic et al. (1999), citado en Buvinic, Morrison y Orlando (2005), señalan que la violencia se define como "el uso o amenaza del uso de la fuerza física o psicológica, con intención de hacer daño" (p. 167), la cual, en cualquiera de sus manifestaciones, ya sea en forma de homicidios, robos, secuestros o violencia intrafamiliar, es uno de los mayores obstáculos para el desarrollo de la región latinoamericana. Al igual que señala la OPS (2002), para Buvinic et al. (2005) la violencia incluye el suicidio y otras conductas autodestructivas y de índole sexual que pueden darse entre extraños, conocidos, e incluso entre familiares.

Para Buvinic et al. (2005) la violencia es un fenómeno complejo, multidimensional y que obedece a factores psicológicos, biológicos, económicos, sociales y culturales, al tiempo que los fenómenos que acompañan el comportamiento violento cruzan las fronteras entre individuo, familia, comunidad y sociedad. Siguiendo con el mismo trabajo, las clasificaciones de la violencia pueden depender de: a) las víctimas, entendidas como grupos poblacionales o contra la propiedad; b) de los agentes violentos, tales como pandillas, narcotraficantes, muchedumbres, movimientos políticos; c) de la naturaleza de la violencia, a saber, física, psicológica, sexual forzada, privación de la libertad, incluyendo el arresto injustificado; d) de la intención de la violencia, la cual puede ser instrumental, es decir, un medio para lograr otros fines, ya sean políticos, económicos, religiosos y sociales, o bien emocional, es decir, causar daño como fin en sí mismo; de acuerdo con el ámbito geográfico, rural o urbano; y, e) de la relación entre la víctima y el agresor, la cual es la social, es decir, entre desconocidos o conocidos sin parentesco, o bien intrafamiliar. 
Los factores de riesgo de la violencia pueden ser de índole: a) personal, tales como factores biológicos, exposición temprana a la violencia, nivel socioeconómico y educacional, abuso del alcohol y drogas; b) del hogar, influenciado por su tamaño y densidad, la estructura, dinámica y normas del hogar, así como antecedentes de violencia en el mismo; y c) de comunidad o social, donde influyen factores tales como la desigualdad, mercados legales e ilegales de armas o drogas, violencia en los medios de comunicación, efectividad de las instituciones de control social, cultural e historias de violencia social en la comunidad (Buvinic et al., 2005).

Para Galtung (1998, p. 15-16) la violencia puede clasificarse en: i) directa, la cual es visible y se refiere a daños físicos, psicológicos o agresiones verbales de una persona o emisor sobre otra, generalmente en relaciones de poder asimétricas; ii) estructural, en la cual no hay una persona concreta que efectúe el acto de violencia, sino que proviene de la estructura social, es decir, es la suma total de todos los choques incrustados en las estructuras sociales y mundiales, que son cementados y solidificados, de tal forma que los resultados injustos, desiguales, son casi inmutables; y, iii) cultural, que se refiere a la suma total de todos los mitos, de gloria, traumas y demás, que sirven para justificar la violencia directa y que legitiman la violencia desde el arte, la religión, el derecho, la ideología, la educación o medios de información. Los rasgos distintivos recaen en que la violencia directa se trata de un evento; la violencia estructural, de un proceso con sus subidas y bajadas; y la violencia cultural es "permanente", dado que permanece esencialmente igual por largos períodos debido a que las transformaciones en la cultura básica son lentas, (Galtung y Fischer, 2013, p. 41). Johan Galtung y Dietrich Fischer (2013) señalan que existe generalmente un flujo causal de la violencia cultural pasando por la estructural y terminando en la violencia directa; no obstante, esta no es única puesto que existen conexiones y flujos causales en todas las direcciones (p. 42).

De manera similar, para Jiménez-Bautista (2012) la violencia cultural es la base donde se sustenta la violencia directa y estructural, pues al conformarse de ideas, valores, normas, tradiciones y lenguajes, el poder actúa e instituye dicha violencia a través de estos instrumentos. Para este autor, es la violencia cultural la que a su vez se transforma en violencia estructural y por último en violencia directa, mientras que como se mencionó, para Galtung (1998, pp. 15-16) son tanto la violencia cultural como la estructural las que se pueden transformar en violencia directa.

Más allá de la violencia estructural y la cultural, autores como Bourdieu (2000) señalan que la violencia simbólica está conformada por instrumentos estructurados y estructurantes del conocimiento, el discurso y su comunicación, que buscan imponer la legitimación del poder y la dominación de una clase sobre otra, donde se monopoliza la construcción de creencias acerca de cómo y por qué se presentan ciertos tipos de acciones por parte de la clase dominante sobre la dominada, perdiendo esta última a su vez el poder para construir símbolos.

$\mathrm{Ni}$ la OPS (2002) ni Buvinic et al. (2005) señalan en sus definiciones o tipologías la violencia contra los animales, la cual no es solo considerada por algunos psicólogos como una precursora de la violencia contra las personas, sino que circunscriben el fenómeno entre seres humanos o entre seres humanos y sus 
pertenencias (López-Ortega, 2011). En todo caso, enmarcándola dentro de los tres grandes tipos de violencia (directa, estructural y cultural) de Galtung (1998), la violencia contra los animales tiene sus raíces en la violencia cultural, que en algunas religiones está determinada por la creencia de la superioridad del ser humano sobre cualquier otro ser vivo en la Tierra, o discusiones sobre si el ser humano está por encima o no de cualquier ser vivo en la cadena alimenticia, o creencias en debate acerca de si los animales tienen o no sentimientos, enmarcándose todo ello en la violencia cultural.

Relacionado con lo anterior, recientemente Galtung y Fischer (2013, p. 34-35) señalaron los efectos visibles e invisibles de la violencia directa en seis dimensiones que se describen en el cuadro siguiente:

\begin{tabular}{|c|c|c|}
\hline \multicolumn{3}{|c|}{ Cuadro 1. Los efectos visibles e invisibles de la violencia directa } \\
\hline Dimensión & Efectos visibles, materiales & Efectos invisibles, no materiales \\
\hline Naturaleza & $\begin{array}{l}\text { Agotamiento y contaminación } \\
\text { Daño a la diversidad y simbiosis }\end{array}$ & $\begin{array}{l}\text { Menos respeto por la naturaleza } \\
\text { no humana, reforzamiento del } \\
\text { concepto "hombre por encima de la } \\
\text { naturaleza" }\end{array}$ \\
\hline Seres humanos & $\begin{array}{l}\text { Efectos somáticos: número de } \\
\text { muertos, heridos, violados, } \\
\text { desplazados }\end{array}$ & $\begin{array}{l}\text { Efectos espirituales: traumas de } \\
\text { duelo, venganza, de odio, adicción, } \\
\text { adicción a la victoria }\end{array}$ \\
\hline Sociedad & $\begin{array}{l}\text { El daño material hacia edificios e } \\
\text { infraestructura }\end{array}$ & $\begin{array}{l}\text { El daño estructural }{ }^{1} \text {, el daño } \\
\text { cultural }^{2}\end{array}$ \\
\hline Mundo & $\begin{array}{l}\text { El daño material hacia la } \\
\text { infraestructura }\end{array}$ & El daño estructural, el daño cultural \\
\hline Tiempo & $\begin{array}{l}\text { Violencia retrasada; minas terrestres } \\
\text { transmiten violencia; genética }\end{array}$ & $\begin{array}{l}\text { Transferencia de estructura, } \\
\text { transferencia de cultura; puntos } \\
\text { kairos }^{3} \text { de trauma y gloria }\end{array}$ \\
\hline Cultura & $\begin{array}{l}\text { Daño irreversible hacia la herencia } \\
\text { cultural humana }\end{array}$ & $\begin{array}{l}\text { Violencia cultural de trauma, } \\
\text { gloria; deterioro de la capacidad de } \\
\text { resolución de conflictos }\end{array}$ \\
\hline
\end{tabular}

Fuente: Galtung y Fischer (2013, p. 34-35).

Notas: 1 . Se refiere a la verticalización de las estructuras organizadas para la violencia, que contienen cadenas ocultas de comandos que con facilidad se convierte en características permanentes de países liberados a través de la violencia. 2. Se trata del rápido crecimiento de la cultura de la violencia para justificar toda violencia directa, con un impacto perdurable. 3. Kairos es una palabra griega que hace alusión "al momento adecuado u oportuno".

Así, vemos que para Galtung y Fischer (2013, pp. 34-35) la violencia contra los animales se enmarca con efectos en la dimensión de la naturaleza, vista como una falta de respeto a la misma que causa como efectos visibles daños a la diversidad, e invisibles, como una falta de respeto a otros seres vivos por ese sentido de superioridad del hombre que ya mencionamos.

Por su parte, Buvinic et al. (2005, pp. 193-200) clasifican los costos socieconómicos de la violencia en: a) directos o monetarios, comprenden el valor de bienes y servicios utilizados en prevenirla, paliar consecuencias en víctimas y procesar a los perpetradores; b) no monetarios, tales como los impactos en la salud a 
través de una mayor incidencia en morbilidad, mortalidad, suicidios, adicciones y desórdenes psicológicos; c) multiplicadores económicos, implican una menor acumulación de capital humano, menor tasa de participación laboral, mayor ausentismo y menores ingresos; y d) multiplicadores sociales, comprenden la transmisión intergeneracional de la violencia a través del aprendizaje, la erosión del capital social, calidad de vida reducida y disminución en la participación social en los procesos democráticos.

Una estimación sobre los costos de la violencia y la inseguridad en México, realizado por el INSYDE, señala que los costos son tanto tangibles como intangibles. Los primeros son todas aquellas actividades relacionadas con la prevención y el castigo de la violencia. Los costos intangibles incluyen aquellos relacionados con la pérdida de calidad de vida de la víctima, cambios en la conducta y traumas personales, pérdida de confianza interpersonal y de capital social a causa del miedo, así como la pérdida de libertad (Magaloni-BID citado en INSYDE, 2014, p. 6).

Los costos tangibles que se han estimado para México según las cifras que cita en su documento el INSYDE (2014), son de 12,3\% del PIB (BID, 2000) o de alrededor de 15\% del PIB según el CEESP (2011). El PNUD (2013) indicó que en 2009 México perdió seis meses en la expectativa de vida de sus habitantes; la Encuesta Nacional de Victimización y Percepción de la Inseguridad (ENVIPE) mostró que el costo de la inseguridad y violencia en los hogares representó un monto de 215,2 mil millones de pesos, equivalente a 5.953 pesos por persona afectada por la inseguridad y el delito (INSYDE, 2014, pp. 28-31).

\section{Antecedentes de las colonias seleccionadas y estrategia metodológica}

La colonia Fomerrey 45, conocida como La Estanzuela, se ubica al sur de la ciudad de Monterrey y cuenta con una población total de 4.479 habitantes (Gobierno del Estado de Nuevo León, 2013a). Se ubica en terrenos federales y casi la mitad de la población vive en la parte alta, en la loma, donde no se cuenta con los servicios de agua, luz, drenaje, ni gas natural. Alrededor de la colonia se han construido fraccionamientos de clase media y media alta, que han cercado a la colonia, haciendo más difícil la llegada y salida de la misma, situación que se agrava en época de lluvias (Vecinos de Fomerrey 45, comunicación personal, 13 agosto de 2013).

Por su parte, la colonia La Alianza se ubica al poniente del municipio de Monterrey, entre las avenidas Abraham Lincoln y San Bernabé. De acuerdo con el Gobierno del Estado de Nuevo León (2013a), en la colonia hay una población total de 242 personas. Sin embargo, en algunos lotes baldíos ha habido intentos de posesionarse e incrementar la población. Existe un Centro Comunitario de Desarrollo Social, "La Alianza" y varias calles aún sin pavimentar (Vecinos de La Alianza, comunicación personal, 27 agosto de 2013).

La tercera colonia analizada, La Independencia, se localiza en el centro de la ciudad de Monterrey, al costado sur del río Santa Catarina y la Avenida Morones Prieto. La colonia La Independencia tiene una población de 228.230 personas (Gobierno del Estado de Nuevo León, 2013b). Casas-García (2010) apunta que la historia de la colonia La Independencia está muy ligada a los primeros asentamientos urbanos cerca del primer cuadro de la ciudad. La colonia era conocida 
como el barrio de San Luisito, debido a la gran cantidad de habitantes llegados del estado de San Luis Potosí desde mediados de 1800. Según Fernández-Areu (2010), además de recibir migración de San Luis Potosí, la colonia cuenta ahora entre sus colonos con personas procedentes de diversos estados, incluyendo grupos indígenas, como los nahuas del municipio de Acaxochitlán, de las sierras del estado de Hidalgo, así como hablantes zapotecos del Istmo oaxaqueño y mayas del sur de Yucatán, entre otros. A pesar de ser un barrio tradicional de la ciudad, esta colonia también se caracteriza por estar en la lista de zonas de atención prioritaria urbana; de las tres colonias aquí analizadas, es la colonia más grande en cuanto a población. La colonia cuenta con un Macro Centro Comunitario de Desarrollo Social, el cual tiene aulas para la enseñanza de diversos oficios, biblioteca, gimnasio, canchas techadas y al aire libre (Vecinos de La Independencia, comunicación personal, 31 agosto de 2013).

La metodología empleada en esta investigación fue cualitativa y se desarrolló en dos etapas. Una primera etapa, consistió en observación planificada y entrevistas preparatorias con vecinos y personal adscrito a centros comunitarios de las tres colonias en estudio. La segunda etapa tuvo como base la realización de talleres participativos con jóvenes; siguiendo los criterios de respeto, pluralidad, libertad de expresión y no imposición. Si bien la inseguridad ha disminuido en los últimos años en el estado de Nuevo León, aún se presentan algunos hechos violentos en las colonias donde se llevó a cabo el estudio, por lo que para proteger la integridad de los vecinos y jóvenes que participaron en la presente investigación se mantienen en anonimato las identidades de los participantes.

Según la Organización Mundial de la Salud (1986, pp. 11-12), la juventud consta formalmente de dos etapas: una, que es la adolescencia, caracterizada por un período de maduración biológica, psicológica y social, representado por la transición entre la niñez y la juventud; y otra, que es propiamente la juventud, la cual es un período de transición entre la adolescencia y la edad adulta. Estas dos etapas son combinadas en una, llamadas indistintamente adolescencia o juventud. Sin embargo, según la OMS (1986, p. 12), la juventud va de los 10 a los 24 años, dividida en tres grupos etarios: de 10 a 14 años; de 15 a 19 años; y de 20 a 24 años. Para fines de esta investigación, por juventud se entiende la población comprendida entre los 12 y los 29 años de edad, según lo estipulado en la Ley del Instituto Mexicano de la Juventud (Cámara de Diputados, 1999).

La división por grupos etarios fue muy útil para el caso de Nuevo León, ya que se ha detectado que muchos niños y adolescentes a veces no son clasificados oficialmente como jóvenes, empero sí enfrentan los mismos problemas, tales como el acoso escolar, depresión, suicidio, pandillerismo, alcoholismo, drogadicción, vandalismo y delincuencia organizada (Hernández de Frutos, Sarabia y Casares, 2002; Scandroglio et al., 2002; Barreira, 2009).

Para la elaboración de la metodología de talleres participativos de previsión social del delito se tomaron en cuenta experiencias realizadas en otros países en contextos similares de violencia entre la juventud, así como experiencias de planeación participativa en otros temas de la agenda pública, como la salud y la educación. Se analizaron metodologías de fuentes como Cabannes (2005), Ministerio de Salud de Argentina (2004), Nirenberg (2006), Korstanje (2009), Krichesky y Flor (2007), ONU (2011), Sánchez-Guerrero (2003), entre otros. 
Se utilizó básicamente la planificación local participativa (PLP) como herramienta metodológica, la cual "implica un proceso continuo de involucramiento en el diagnóstico, programación de acción, ejecución y evaluación, que permite a las personas y comunidades conocer su realidad, a la vez que sentirse capaces de actuar sobre ella y transformarla" (Ministerio de Salud de Argentina, 2004, p. 3). El diagnóstico consta de cinco etapas: mapeo de actores; diagnóstico participativo; presupuesto participativo; planeación y desarrollo de proyectos y acciones; evaluación e institucionalización de proyecto y acciones exitosas.

El mapeo de actores consistió en identificar y establecer contacto con líderes de las colonias, jóvenes que a su vez forman parte de otros grupos dentro de su comunidad, por ejemplo, miembros de un equipo deportivo así como personal de los centros comunitarios. El diagnóstico participativo se constituyó siguiendo los lineamientos de la Secretaría de Gobernación (2013) en dos momentos: primero, en la emisión de la convocatoria pública y plural para participar en el taller, en un segundo momento, se llevó a cabo el taller el cual permitió que los asistentes se conocieran, identificaran los problemas de sus comunidades y llegaran a un consenso sobre estos. Las herramientas utilizadas durante el taller fueron la lluvia de ideas para identificar los problemas; la priorización de estos a través de la votación directa en conjunto atendiendo a los criterios de: a) que atienda a la necesidad central (prevención de la violencia), b) que tenga aplicación a toda la comunidad, c) que el número de beneficiarios sea el mayor posible, d) que atienda a colectivos identificados dentro de los jóvenes (Francés y Carrillo, 2008); realizar un árbol de problemas para conocer las posibles causas y efectos así como las soluciones a través de proyectos enfocados hacia y para los jóvenes.

En el caso de la colonia Fomerrey 45 - La Estanzuela, el taller se llevó a cabo en la propiedad de uno de los vecinos, a falta de un lugar público techado. En total, en el taller participaron 18 jóvenes entre 12 y 28 años de edad. La edad promedio fue 17 años; del total de participantes, $83 \%$ fueron hombres y $17 \%$ mujeres. Respecto a la ocupación, $39 \%$ no estudia ni trabaja, otro $39 \%$ estudia, $17 \%$ trabaja y el resto tiene como ocupación el hogar. De entre los asistentes, $17 \%$ manifestó tener hijos.

En La Alianza, el taller se llevó a cabo en el Centro Comunitario de la colonia. Asistieron 32 jóvenes, de 12 a 26 años, con una edad promedio de 16 años. Del total, $30 \%$ fueron mujeres y el resto varones. Casi todos manifestaron estar estudiando, salvo dos personas, una que trabaja y una ama de casa.

En la colonia La Independencia, el taller se llevó a cabo en el Centro Comunitario de Desarrollo Social "Bicentenario de la Independencia", un macrocentro inaugurado en 2011. El taller contó con la presencia de 15 jóvenes, de los cuales $33 \%$ se trató de mujeres. La edad promedio del grupo fue 18 años. Casi todos manifestaron estudiar salvo una persona.

\section{Resultados de los talleres con jóvenes}

En una primera instancia, se les preguntó de manera abierta a los jóvenes sobre las principales problemáticas que viven día a día en su colonia. Posteriormente, se les pidió que reflexionaran sobre los significados de la violencia social, sus causas y efectos. 


\subsection{Principales problemas enfrentados por la juventud}

En la colonia Fomerrey 45 - La Estanzuela, las y los jóvenes observaron 82 problemas, de los cuales las peleas ocupan el porcentaje más alto, con $18 \%$ de las menciones. Los tipos de peleas que los jóvenes observan incluyen: peleas sin mayor detalle (33\%); entre pandillas (27\%); en los partidos de fútbol (13\%); en las calles (13\%); causadas por el alcohol (7\%); y entre la familia (7\%). Sobre los detalles de las peleas, los jóvenes manifestaron: "No saben perder en el fútbol y luego luego (sic) se van a los golpes". "Hay muchos que son abusivos". "Se pelean por las esposas".

Después de las peleas, el siguiente problema en importancia es la inseguridad, con $16 \%$ de las menciones. Los hombres indicaron que se sienten más inseguros que las mujeres:

Los malitos [delincuentes] te llevan y te arrastran un rato y luego te dejan por ahí. Buscan más a los hombres para meterlos a la delincuencia y no a las mujeres. Y si no quieres, te llevan a la fuerza. Sino llegan dos o tres changos [delincuentes] en una camioneta y lo echan para arriba y ya no vuelves a ver a la persona. Se han llevado de aquí como 30, 60 o 100. Ya no se han vuelto a ver. Unos amigos estaban en la esquina esperando el camión para irse a trabajar en la mañana y de ahí se los llevaron. Andaban aquí día, tarde y noche y no sabías si salías y regresabas (Talleres con jóvenes, comunicación personal, 2013).

Enseguida están los robos, con $12 \%$ de las menciones. "Hay muchas ratas [ladrones] aquí", mencionó uno de los jóvenes. El problema que sigue en importancia según lo que observan los jóvenes en la colonia es el relacionado con las drogas y los drogadictos, con $12 \%$.

Se les preguntó si las drogas son buenas o malas, si causan problemas o los solucionan, a lo que contestaron: " $E$ Es buena, te relaja, te sube al avión! Se consigue yerba [marihuana], piedra, cristal, pastillas. La gente usa la yerba para trabajar, te ayuda, además es más natural que el tabaco".

Casi igual en importancia está el problema de "los borrachos" y el alcoholismo, con $11 \%$ de las menciones. "Mis vecinos toman cerveza, pero se alocan y se agarran a madrazos [golpes]", manifestó un joven.

Las balaceras y el pandillerismo ocupan $5 \%$ de las menciones cada una. Cuestiones como la falta de trabajo, la prostitución y la Fuerza Civil (Policía estatal) representaron $2 \%$ de las menciones cada una.

Cabe destacar la relación que tienen los jóvenes con la autoridad policial, específicamente con la Fuerza Civil del Gobierno del Estado de Nuevo León. A continuación se muestran los comentarios que al respecto hicieron varios jóvenes:

¡La Fuerza Civil! Aquí se la viven, los corrieron de aquí, ya no tienen cuartel, pero vienen todos los días. Está bien que pasen, pero se pasan de vivos. Pasan y te dan sopapos [golpes en la cabeza], te trasculcan [revisan]. Es por la gente que chismea, nos reportan. Nada más quieren andar manoseando gente. Te quitan el dinero. Una noche andaba con mi primo y mi hermano y pasaron y luego luego (sic) nos pararon, te encañonan y te dicen que a la otra te revientan la cabeza. Andábamos en un taxi y nos revisaron, pero nada más íbamos por unas cocas [los demás se ríen, dicen que más bien iban por cervezas]. A muchos vecinos no les gusta vernos en bolita, que andamos jugando futbol y nos reportan, pero si los vecinos ven a los que fuman 
mota [marihuana], ni le hablan a la Fuerza. Nada más les hablan cuando les conviene. A los que deben revisar no los revisan. (Talleres con jóvenes, comunicación personal, 2013).

Otros problemas identificados por los jóvenes en su colonia, con $1 \%$ de las menciones cada uno, fueron: asesinatos, vandalismo, delincuencia, corrupción, falta de servicios, falta de luz, falta de cultura, problemas entre la familia, que la gente tire basura en las calles, los vecinos chismosos y la música alta.

En la colonia La Alianza, los jóvenes mencionaron 179 principales problemas observados en el día a día, los que tienen que ver con peleas son el $16,76 \%$. Entre los tipos de peleas mencionadas destacan las peleas entre vecinos, seguidas por las peleas entre pandillas, las peleas entre miembros de diferentes colonias y las que se suscitan en la escuela. Los jóvenes mencionaron que las peleas se dan en: "La secundaria; entre pandillas; $y$ en los partidos de futbol". Entre las razones de las peleas dijeron que eran: "porque se emborrachan los vecinos; por los territorios; por viejas rencillas; porque hay gente que se cree mucho y vienen muy acáb (sic)". Agregaron que se pelean entre jóvenes de las diferentes colonias y calles y "se agarran a riscasos [pedradas]" (Talleres con jóvenes, comunicación personal, 2013).

El siguiente problema con más menciones, $14 \%$, fue la contaminación y la basura en las calles. Lo anterior coincide con lo observado en la colonia por el equipo de investigación.

El tercer problema observado con más menciones es el relativo a las drogas, con $11,7 \%$. Preguntados acerca de cómo se presenta el problema de las drogas en su colonia, los jóvenes mencionaron que la droga se consume en los parques y que las drogas más utilizadas son la mariguana, el Resistol 5000, el tolueno, el tíner, la cocaína, la piedra, así como las pastillas. Sobre qué tan complicado es conseguir las pastillas u otras drogas y su precio, dijeron "es muy fácil, vas a la esquina o al callejón y te las venden. La mota (mariguana) la venden en bolsitas de 50 pesos, el tolueno vale desde 20 pesos o lo que tú quieras y las pastillas valen 80 pesos". Se les preguntó si creen que las drogas son buenas o malas, por qué la gente se droga y si te dan o te quitan problemas. Sus respuestas fueron: "Las drogas son malas porque te hacen daño en los pulmones, te enjoroban [encorvan], te hacen daño en el cuerpo / La gente se droga por los problemas, para borrar los problemas, terminar con ellos, se olvidan de todo, ya lo he hecho / Las drogas te dan más problemas" (Talleres con jóvenes, comunicación personal, 2013).

El siguiente problema más observado que se reportó fue el pandillerismo, con $11,7 \%$. Respecto a los robos, estos se mencionaron en $8,9 \%$ de los casos. Al respecto, los jóvenes dijeron que: "Te roban la ropa, los tenis / a mi papá le robaron la pila del carro hace una semana / Hay robos en las escuelas y las casas / Se roban las lámparas de las plazas".

Otro problema fue el alcoholismo, pero más que mencionarlo en general, los asistentes se refirieron a "los borrachos" como su principal preocupación. Dijeron que es más la gente que anda tomando en la calle que la que se droga y que eso causa buena parte de las peleas.

6 En el argot callejero, la expresión "vienes muy acá" se refiere a llegar con mucha valentía, mientras que "creerse muy acá" significa creerse mucho, es decir, ser pedante. 
Un problema más es el del maltrato animal, con 3,9\% de los casos. Los jóvenes señalaron ver perros con sarna en la calle, así como atropellos premeditados de perros. Con igual porcentaje fue mencionado el problema de la violencia hacia las mujeres, violaciones en casas abandonadas y acoso sexual. Las mujeres mencionaron: "Hay mucho monte y ahí se meten a drogar / existe abuso sexual / nos molestan / nos acosan y nos persiguen".

El resto de los problemas observados obtuvieron porcentajes individuales de menciones menores a 3,3\%, cada uno son: bullying, balaceras, falta de luz mercurial, delincuencia, maltrato a parques, vandalismo, lotes baldíos, violencia intrafamiliar, maltrato y golpes a personas, violencia, grafiti, malas compañías, muerte, injusticias, desnutrición infantil, niños que no estudian, alta velocidad de autos, discriminación, fumadores, indigencia.

Por último, los jóvenes de la colonia La Independencia manifestaron una lista de 92 problemas en su colonia, que pueden dividirse en 26 problemas comunes. El problema que resultó con más menciones, fue la inseguridad, con 6,7\% del total. Se les pidió que describieran la inseguridad que vivían, a lo que respondieron: "Es más peligroso por la noche". "La parte de arriba es más peligrosa, en la loma". "Te persiguen, te molestan, te roban". A la pregunta ¿Para quién es más peligroso, para los hombres o las mujeres?, respondieron: "Es más inseguro para las mujeres (dijo un hombre), porque ellas no se pueden defender y los hombres sí". Una mujer agregó: "Vas caminando y te faltan al respeto. No se acercan, pero te faltan al respeto. Pueden ser muchachitos de 12 o 13 años que salen de la secundaria, o un señor que está por ahí tomando caguamas [cerveza tamaño grande] en una esquina. La mayoría de las veces se quedan en palabras, pero a veces te siguen".

Los problemas de drogas resultaron con 5,6\% de las menciones. Interrogados acerca de las drogas disponibles en su colonia, mencionaron que son: mariguana, cocaína, píldoras (éxtasis), inyecciones, la piedra, tolueno, Resistol 5000 y peyote. Los jóvenes indicaron que es muy fácil conseguir drogas en su colonia. Sobre los costos aproximados dijeron que la dosis de mariguana vale, dependiendo del tamaño, entre 20 y 50 pesos, mientras que el tolueno lo venden desde 5 pesos, en cualquier ferretería, siempre y cuando lo compren mayores de edad. A la pregunta de si las drogas son buenas o malas, los jóvenes contestaron: "Son malas, es una mafia". "Dañan tu salud, la memoria, el cerebro". "Dañan tu integridad, si alguien se droga, después puede llegar a hacer otras cosas para conseguir una dosis, como robar".

El siguiente problema observado por los jóvenes fue el maltrato infantil, con $5 \%$ de las menciones. Los jóvenes señalaron que es común ver que "las señoras maltratan mucho a sus niños, les gritan las peores cosas a los niños, o los traen descalzos, los agreden verbalmente desde chiquitos".

El alcoholismo fue mencionado en 4,5\% por los jóvenes. Mencionaron que es común ver gente tomando caguamas en las calles. El alcohol, dijeron, "causa riñas, por mi casa hay dos pandillas y se pelean cuando andan tomados".

La violencia fue mencionada como problema en sí mismo 3,9\% de las veces; los robos y asaltos, 3,4\%; las peleas, falta de luz mercurial, violencia intrafamiliar y secuestros 2,2\% cada uno. Sobre los secuestros mencionaron que: "Antes 
secuestraban a mujeres y jóvenes, doctores, a los que se encontraran, para pedir dinero". Dijeron que ya han disminuido.

Otro problema mencionado fue el de las balaceras, con 1,7\%. Dijeron que "las balaceras han disminuido, pero aún se escuchan, a todas horas, más en la madrugada, en la parte de arriba". Las pandillas fueron mencionadas con 0,6\%. "Ya casi no hay pandillas, solo cárteles (de drogas)", dijeron. El resto de los problemas mencionados fueron: maltrato animal, acoso sexual, vandalismo y grafiti, falta de respeto entre las personas, vagancia, pandillerismo, delincuencia organizada, maltrato y golpes a personas, muertes, falta de estudios, violencia verbal, violencia entre parejas, calles en mal estado, y tabaquismo.

Muchos de los problemas mencionados por las y los jóvenes en las tres colonias son comunes, como las peleas, inseguridad, robos, pandillerismo, drogadicción, alcoholismo, violencia intrafamiliar, violencia contra la mujer y vandalismo, mientras que otros como el descuido de espacios públicos y el maltrato animal son específicos de algunas de las colonias. Muchas de las problemáticas están directamente relacionadas con el concepto de violencia, sus tipologías directa, estructural y cultural; así como los flujos causales de estas. El resto de los problemas identificados guardan una estrecha relación con los factores promotores de violencia, como lo es la falta de infraestructura pública en las colonias. En la siguiente sección, se analizan los significados que de la violencia tienen las y los jóvenes que participaron en el estudio.

\subsection{Significados de violencia entre las juventudes}

Se les preguntó a los jóvenes qué significa para cada uno de ellos la violencia social y posteriormente se les pidió que formaran equipos, les pusieran un nombre y llegaran a un acuerdo grupal de los significados de la violencia. A continuación se muestran los resultados de los talleres en las tres colonias.

Cuadro 2. Significado de la violencia para los jóvenes

\begin{tabular}{lll}
\hline Colonia & \multicolumn{1}{c}{ Equipo } & \multicolumn{1}{c}{ Significados de la violencia } \\
\hline & Los DJ & $\begin{array}{l}\text { Matar, robar, agredir a una persona por sus gustos, abusar } \\
\text { sexualmente, golpear a alguien, ofender. }\end{array}$ \\
& $\begin{array}{l}\text { Es una acción inoportuna que ciertas personas causan por } \\
\text { consecuencia del alcohol, drogadicción y muchas sustancias } \\
\text { más que son dañinas para la salud; muchos de los vecinos } \\
\text { viven a diario la violencia social. }\end{array}$ \\
& Los Fresitas & $\begin{array}{l}\text { Abusar de una persona, bullying, peleas en la colonia y entre } \\
\text { familias, balazos, discutir entre personas, problemas en } \\
\text { pandillas, matar. }\end{array}$ \\
& Los ¡Sallayin! & $\begin{array}{l}\text { Racismo, faltar el respeto a las personas, peleas entre amigos } \\
\text { o entre familia, robos en la colonia o en las tiendas, que los } \\
\text { de San luis son muy peleoneros, faltarle al respeto a la gente } \\
\text { discapacitada. }\end{array}$ \\
\hline
\end{tabular}


Cuadro 2. Significado de la violencia para los jóvenes

\begin{tabular}{|c|c|c|}
\hline Colonia & Equipo & Significados de la violencia \\
\hline \multirow{8}{*}{ 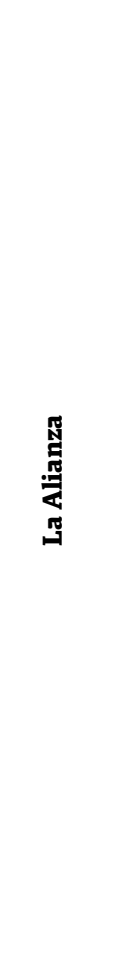 } & $\begin{array}{l}\text { Los Chofris Locotes } \\
\text { de PSB }\end{array}$ & $\begin{array}{l}\text { Maltrato hacia otra persona; ofensas; insultos; groserías; } \\
\text { acosamiento (sic) sexual, verbal, físico, psicológico. }\end{array}$ \\
\hline & $\begin{array}{l}\text { ¡Fricky, Locos! } \\
\text { Negros }\end{array}$ & $\begin{array}{l}\text { Que la comunidad no hace nada por evitar el bullying y ayudan } \\
\text { a generar más bullying. }\end{array}$ \\
\hline & Aguaciel & $\begin{array}{l}\text { El bullying, cuando muchas personas agreden a alguien } \\
\text { indefenso, o verbalmente, o son racistas; violencia verbal: } \\
\text { cuando amenazan, te ponen apodos por tu forma de ser; es un } \\
\text { acto indebido hacia otras personas. }\end{array}$ \\
\hline & $\begin{array}{l}\text { La Liga de la } \\
\text { Justicia }\end{array}$ & $\begin{array}{l}\text { Violar las normas sociales que hay en tu comunidad, cuando } \\
\text { golpean a alguien en la calle, cuando insultan a alguien por la } \\
\text { calle, peleas entre amigos, discriminación. }\end{array}$ \\
\hline & $\begin{array}{l}\text { Los Guerreros (La } \\
\text { Alianza) }\end{array}$ & $\begin{array}{l}\text { Los más grandes sienten que tienen más poder sobre los } \\
\text { más chicos, se golpean e insultan entre ellos mismos, hacen } \\
\text { bullying en las escuelas; maltrato contra las mujeres y acoso; } \\
\text { insultos; racismo. }\end{array}$ \\
\hline & $\begin{array}{l}\text { El Número } 7 \text { de la } \\
\text { Suerte (La Alianza) }\end{array}$ & $\begin{array}{l}\text { Insultar, bullying, pelear, maltratar a las mujeres, maltrato } \\
\text { infantil, abuso sexual, discriminación, maltrato animal, } \\
\text { racismo; todo aquello que pase por encima de los derechos de } \\
\text { los demás. }\end{array}$ \\
\hline & Las Pequezh & $\begin{array}{l}\text { Insultar a los chavos y chavas, bullying, discriminación a las } \\
\text { chavas. }\end{array}$ \\
\hline & Los Diablos & $\begin{array}{l}\text { Maltrato hacia otras personas, bullying, agredir niños y niñas } \\
\text { menores, respetar a los mayores si quieres que te respeten, } \\
\text { amenazar a la gente, maltratar a la gente. }\end{array}$ \\
\hline \multirow{4}{*}{ 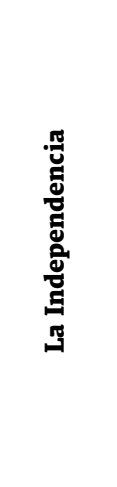 } & No a la Violencia & $\begin{array}{l}\text { Violencia entre la familia: maltrato, golpes; violencia verbal: } \\
\text { ofensas, gritos, regaños; violencia física: golpes; abusos: entre } \\
\text { las mujeres, golpes a ellas y violaciones [sic]. }\end{array}$ \\
\hline & $\begin{array}{l}\text { Las Mujeres y un } \\
\text { Colado }\end{array}$ & $\begin{array}{l}\text { Agresión y falta de respeto hacia los valores, integridad física } \\
\text { y/o moral de una persona o comunidad, creándose un círculo } \\
\text { vicioso o una cadena interminable de abusos y agresiones } \\
\text { derivada de la ignorancia, la intolerancia y la frustración. }\end{array}$ \\
\hline & 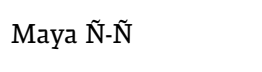 & $\begin{array}{l}\text { Abusos físicos y emocionales; balaceras; robos; un problema en } \\
\text { la sociedad. }\end{array}$ \\
\hline & Los Sexys & $\begin{array}{l}\text { Balaceras, secuestros, cuando se metían a las casas, cuando } \\
\text { incendiaban las casas, pedían cuotas, amenazas, violencia } \\
\text { verbal. }\end{array}$ \\
\hline
\end{tabular}

Fuente: Elaboración propia con base en el taller de diagnóstico participativo en las colonias Fomerrey 45-La Estanzuela, La Alianza y La Independencia.

Nota: Los talleres se realizaron en los días 13 y 17 de agosto y 20 septiembre de 2013 en las colonias Fomerrey 45-La Estanzuela, La Alianza y La Independencia, respectivamente.

Según las tipologías de Galtung (1998), los tipos de violencia definidos por los jóvenes de la Fomerrey 45-La Estanzuela fueron: la directa, que incluye daños físicos como matar, golpear, abusar sexualmente, peleas, discusiones; así como 
daños psicológicos como bullying, ofender, agredir a alguien por sus gustos, faltar al respeto a los mayores y a los discapacitados. Siguiendo con las tipologías de Galtung (1998), también se percibe en las definiciones la violencia estructural como racismo, discriminación y violencia social, aunque los jóvenes no ahondaron en lo que significaba para ellos esto último. Según la concepción de la violencia en relaciones de poder asimétrico Galtung (1998), esta se expresa principalmente por el abuso sexual contra mujeres, es decir, el poder del hombre sobre la mujer, así como el abuso de los niños más fuertes contra los más débiles en fenómenos como el bullying o contra personas con discapacidad.

En lo que respecta a las tipologías de Buvinic et al. (2005), los jóvenes de la colonia Fomerrey 45-La Estanzuela señalan los daños a la propiedad, tales como los robos a personas y comercios. Con respecto de quién ejerce la violencia, esta se da tanto entre extraños, como entre conocidos, vecinos, amigos, compañeros de escuela y familiares que pelean entre ellos. Si bien ni Galtung (1998) ni Buvinic et al. (2005), ni la OPS (2002) mencionan como categoría el lugar donde se ejerce la violencia, se destaca que los jóvenes de Fomerrey 45-La Estanzuela perciben que la violencia se presenta principalmente en el hogar, las calles, la escuela y los comercios, estos últimos por los robos.

Por su parte las y los jóvenes de la colonia La Alianza perciben la violencia de forma directa, tomando los mismos modelos de Galtung (1998) como daños físicos: golpes, peleas, maltrato y abuso sexual contra las mujeres, maltrato infantil, así como maltrato animal. Respecto de los daños psicológicos se percibieron: amenazas, ofensas, insultos, groserías, acoso sexual, verbal, psicológico y bullying. Siguiendo con las tipologías de Galtung (1998), también se percibe violencia estructural como racismo y discriminación. Resalta la violencia en relaciones de poder asimétrico descrita por Galtung (1998) en las expresiones de que "los más grandes sienten que tienen más poder sobre los más chicos", "agredir niños y niñas menores" o "maltrato contra las mujeres y acoso". En lo que respecta a las tipologías de Buvinic et al. (2005), la violencia se da tanto entre extraños, como entre amigos y compañeros de escuela. Destaca en esta colonia el maltrato animal entre los significados que para la juventud tiene la violencia. Como ya se mencionó, ni la OPS (2002) ni Buvinic et al. (2005) ni Galtung (1998) señalan en sus definiciones o tipologías la violencia contra los animales, la cual es considerada por algunos psicólogos (López-Ortega, 2011) como precursora de la violencia contra las personas.

Respecto de la colonia La Independencia, los tipos de violencia directa física descritos por los jóvenes, según la tipología de Galtung (1998), son los golpes, violaciones a mujeres, abusos físicos y balaceras, mientras que la violencia directa psicológica está relacionada con: ofensas, gritos, regaños, falta a la integridad moral de las personas o comunidades, abusos emocionales, amenazas, secuestros y el cobro de cuotas a negocios por parte del crimen organizado, lo cual, de no cumplirse, se convierte en violencia física. Respecto a la definición de violencia como relación de poder asimétrico de Galtung (1998), destaca en esta colonia la violencia contra las mujeres, derivada en varias de sus más graves expresiones, como es la violación y agresiones físicas. Según las tipologías de Buvinic et al. (2005), las y los jóvenes señalan graves daños a la propiedad, como el incendio de viviendas por parte del crimen organizado. 


\subsection{Causas de la violencia entre las juventudes}

En la colonia Fomerrey 45 - La Estanzuela, las y los jóvenes mencionaron 66 posibles causas de la violencia que agrupadas en temas comunes dan los siguientes porcentajes: chismes o falta de comunicación y apoyo entre padres e hijos (18,18\%); drogas (16,67\%); alcohol (10,61\%); medios de comunicación, por los anuncios de cerveza o programas de televisión como los de Laura Bozo (9,09\%); problemas familiares por falta de dinero en la casa para comer o porque los jóvenes llegan tarde (9,09\%); sentirse más fuerte que otra persona (9,09\%); problemas en la sociedad y entornos problemáticos (9,09\%); y el porcentaje restante por otras causas tales como por locos, por baja autoestima, por celos, por falta de cultura, falta de dinero o de trabajo y por herencias.

Según la tipología de Buvinic et al. (2005) que divide a las causas de la violencia en factores personales, del hogar, comunitarios, culturales, mediáticos, atribuibles a acción o inacción del Estado y sociales, resalta el hecho de que las y los jóvenes de la colonia Fomerrey 45 - La Estanzuela tienen presentes en conjunto todos los factores anteriores, incluso la influencia de los medios de comunicación en la normalización de la violencia. Siguiendo a Galtung (1998) y Galtung y Fischer (2013), el flujo causal puede entenderse desde la violencia cultural hacia la estructural y evidenciarse a través de la violencia directa. Esto es, tanto los programas televisivos como los anuncios de bebidas alcohólicas permean hacia las estructuras sociales, donde se hace evidente que promueve y genera violencia, dado que ejemplifica comportamientos, actitudes y valores que desembocan en acciones violentas concretas.

En el taller participativo en la colonia la Alianza las y los jóvenes identificaron en conjunto 40 causas de la violencia, que fueron clasificadas en temas comunes, quedando de la siguiente forma: falta de vigilancia de la Policía (12,5\%), porque la gente la permite (12,5\%); peleas entre pandillas (10\%); aprovecharse de los menores o bullying (10\%); por estar acostumbrados a la misma violencia, a tomarla como un juego (10\%); falta de empatía y sensibilidad (7,5\%); por el racismo y discriminación de la gente (7,5\%); por acciones de la Fuerza Civil (5\%); por no saber respetar a los mayores (5\%); porque se vive violencia intrafamiliar (5\%); y el resto debido a otras causas como las drogas, robar y el vandalismo.

Utilizando la misma tipología de Buvinic et al. (2005), en la colonia La Alianza se observa que los factores precursores de la violencia tienen que ver con la falta de acción del Estado, expresada tanto por medio de una vigilancia débil por parte de la Policía, como de manera activa, siendo la Policía el sujeto activo de la violencia hacia los jóvenes, que expresan que "la Fuerza Civil a veces te lleva a la secundaria, pero a veces te quitan el dinero, te quitan la ropa y te la avientan a la avenida. La Fuerza Civil a veces es buena y a veces es mala, te ponen a hacer sentadillas porque estás en las esquinas y no debes estar en las esquinas". Otro resultado interesante encontrado en esta colonia tiene que ver con la normalización de la violencia como causa de la misma. Asimismo, se hizo evidente la violencia cultural, expresada en discriminación y racismo.

En la colonia La Independencia se mencionaron 70 causas que, organizadas por categorías o temas comunes, se muestran a continuación: falta de atención de padres a hijos, escasa educación, valores y desintegración familiar (15,7\%); 
bullying y maltratar a los otros, la cual es más bien un efecto o tipo de violencia y no tanto causa (14,3\%); drogas (14,3\%); alcoholismo (7,1\%); intimidación hacia otros y violencia familiar (7,1\%); falta de policías (7,1\%); carencias económicas en el hogar que hace que la gente se moleste o esté dispuesta a hacer cosas ilegales (5,7\%); estar en pandillas ( $4,3 \%$ ): la frustración de las personas que las hace ser agresivas (2,9\%); tratar de ser "geniales" (2,9\%); falta de comunicación (2,9\%); y con 1,4\% cada una, las siguientes cuestiones: cárteles de la droga, por no querer trabajar con los carteles, inseguridad, por culpa de la sociedad, por los videojuegos violentos, porque los jóvenes no quieren trabajar, por intolerancia, porque las personas optan por defenderse solas, por querer golpear, asaltar, por no tomar buenas decisiones y por causa de las armas.

Tomando la tipología de Buvinic et al. (2005), se observa un fuerte componente de causas atribuibles a la dinámica de los hogares y a causas sociales, como la falta de ingresos, así como a factores personales impactados por el entorno, como la frustración, acceso a alcohol, drogas y armas, y aquellas atribuibles a la inacción del Estado, tales como la falta de policías, presencia de carteles del crimen organizado y armas. Aunque con pocas menciones, resulta interesante que las nuevas tecnologías como los videojuegos violentos sean mencionados como causas de la violencia. Nuevamente en esta colonia se hace evidente el flujo de causas de la violencia, desde la violencia directa pasando por la estructural hasta la violencia cultural y viceversa.

Es interesante cómo los flujos de violencia directa, estructural y cultural surgen y se retroalimentan en cada una de las colonias. De modo tal, que la violencia directa es normalizada y vista como usual, esto es, ha permeado de tal modo que los valores y creencias se han modificado. Asimismo, se puede observar por las respuestas de las y los jóvenes cómo la violencia genera más violencia.

\subsection{Efectos de la violencia entre la juventud}

Como una fase del taller, se les pidió a los jóvenes que ahondaran en los efectos de la violencia, más allá de las manifestaciones de la violencia misma. Si bien en algunas ocasiones se confundían ambas cuestiones, en otros casos hubo resultados interesantes.

En el caso de la colonia Fomerrey 45 - La Estanzuela, en total hubo 66 efectos señalados por las y los jóvenes que, agrupados por similitud, fueron: aspectos emocionales tales como sentimientos de tristeza, desilusión, miedo, enojos, odio, problemas psicológicos y suicidios (22,7\%); que la violencia genera más violencia (15,1\%); violaciones, robos, muertes, balaceras y secuestros (12,1\%); discusiones y malos entendidos (9,1\%); separaciones entre amigos (7,6\%); que la familia se separe (6,1\%); maltratos en la familia (3,0\%); y el resto otros efectos.

En lo que respecta a la colonia La Alianza, se reportaron un total de 74 efectos identificados por las y los jóvenes. Al igual que en el caso anterior se confunden los efectos con la violencia en sí misma, siendo los resultados los siguientes: peleas, pleitos, discusiones y descontento entre vecinos $(20,3 \%)$; divorcios y separaciones de parejas (12,2\%); que la gente se drogue (12,2\%); muertes $(12,2 \%)$; cuestiones psicológicas como falta de amor, temor en la gente, inseguridad interna y odio (9,5\%); inseguridad (9,5\%); conflictos entre personas (4,1\%); efectos 
del acoso y bullying (4,1\%); tener enemigos (4,1\%); violencia contra uno mismo $(1,4 \%)$; y el resto otros efectos.

Por último, en la colonia La Independencia se reportaron 77 posibles efectos de la violencia agrupados en: aspectos psicológicos tales como malestar, sentimientos de rencor, frustración, intranquilidad, sentido de inseguridad, tristeza, miedo, baja autoestima, ansiedad, traumas, depresión y suicidios (20,8\%); asesinatos (18,2\%); peleas (14,3\%); círculo vicioso y traspaso intergeneracional de la violencia $(7,8 \%)$; incremento en alcoholismo y drogas $(5,2 \%)$; desintegración familiar (2,6\%); y el resto otros efectos.

Así, en las tres colonias donde se llevaron a cabo los talleres con jóvenes, podemos concluir que los efectos percibidos de la violencia tienen varias categorías, siendo las más relevantes las psicológicas en las dimensiones ya mencionadas, la desintegración familiar, de círculos de amigos y comunidades, así como la reafirmación de la violencia y el incremento en adicciones. Si bien los autores analizados en el marco teórico señalan las dimensiones de la violencia, no abundan en los efectos de la misma sobre las personas, sus comunidades y la sociedad en general, por lo que se considera que con este análisis a partir del trabajo de campo contribuimos a la comprensión y categorización de dichos efectos. Es interesante que los jóvenes no hayan señalado los costos en pérdida de vidas, así como tampoco los costos económicos que la violencia impone a las familias y a sus comunidades.

\subsection{Proyectos comunitarios para prevenir la violencia entre las juventudes}

La última fase del taller participativo con jóvenes consistió en proponer ideas de proyectos que contribuyeran a reducir la violencia. Estos proyectos se debían basar en atacar las causas de la violencia y una vez consensuados, debía priorizarse a través de la votación simple. Vale la pena señalar que estos proyectos no estuvieron enfocados a atacar los problemas estructurales de sus comunidades, esto es, acceso a servicios básicos en sus viviendas, acceso a empleo y servicios educativos, sino que se enfocaron a aquellos proyectos destinados a aumentar el capital social haciendo uso de los servicios e infraestructura con la que cuentan a través de la participación activa de los jóvenes. A continuación se presentan dichas ideas.

En la colonia Fomerrey 45 - La Estanzuela, las y los jóvenes propusieron los siguientes proyectos: 1 . enseñanza de oficios; 2 . actividades para disminuir la violencia intrafamiliar; 3. cursos de música; 4 . curso para disminuir el consumo de drogas; 5. curso para disminuir el consumo de alcohol.

Por su parte, las y los asistentes al taller de la colonia La Alianza propusieron: 1 . actividades de prevención sobre el consumo de alcohol; 2. enseñanza de oficios; 2 . proyectos deportivos; 3. prevención sobre el consumo de cigarros; 4. prevención sobre el consumo de drogas; 5 . clases de rap; 6 . hacer un pentatlón; 7. más cursos de break dance (ya se imparten en el centro comunitario de la colonia).

Por último, las y los jóvenes en La Independencia propusieron: 1. construcción de una piscina en los terrenos del centro comunitario (otros centros 
comunitarios de la ciudad sí cuentan con ellas); 2. talleres de prevención sobre drogas, alcoholismo y violencia intrafamiliar; 3. torneos deportivos; 4. talleres de arte; 5 . organización de empresas comunitarias; 6. mercado de artesanías y manualidades para venta y promoción; 7. paseos culturales a museos, teatros, etc.; 8. centro de alcohólicos anónimos; 9. vestidores en gimnasio existente en el centro comunitario; 10. mercado de trueques de manualidades o productos que la gente produce.

Estos proyectos buscan combatir las diversas causas de la violencia directa identificadas y evaluadas por ellos mismos. Los proyectos fueron propuestos y evaluados por los jóvenes participantes en los talleres, tomando en consideración los recursos con los que cuenta su comunidad, el capital social por medio de asociaciones de vecinos, así como el aprovechamiento de la infraestructura y actividades que se llevan a cabo en los centros comunitarios en dos de las tres colonias analizadas.

\section{Reflexiones finales}

Los jóvenes de las tres colonias participantes en los talleres enfrentan una serie de problemas cotidianos que condicionan sus posibilidades de tener un desarrollo integral y, por lo tanto, de aprovechar su máximo potencial y con ello mejorar su bienestar.

Los principales problemas observados por los jóvenes están relacionados con formas de violencia directa y estructural, como son las peleas, la inseguridad, los robos, la violencia intrafamiliar y violencia contra la mujer, las drogas, principalmente las de fácil acceso como el tolueno y la mariguana, el alcohol, las balaceras, el pandillerismo, la falta de infraestructura, mantenimiento y equipamiento adecuado en las colonias, la falta de empleo y los malos tratos que reciben por parte del cuerpo policiaco de Fuerza Civil, lo que revela la necesidad de revisar los protocolos de actuación de la Policía en su relación con la población civil, particularmente con los jóvenes, así como mejorar su capacitación respecto a los derechos humanos.

Respecto a la violencia, esta se presenta principalmente en el hogar, las calles, las canchas deportivas y la escuela, este último lugar con fuertes problemas de acoso escolar o bullying. La violencia es infringida por los padres de familia, las pandillas, los narcotraficantes, los alcohólicos, los drogadictos, la Fuerza Civil, los compañeros de clase, los amigos y los vecinos. La violencia se manifiesta en golpes, robos, maltratos verbales y psicológicos, acoso sexual, violaciones y asesinatos. Algo interesante en esta investigación y no previsto en estudios sobre el tema como el de Buvinic et al. (2005), Galtung (1998), Jiménez-Bautista (2012) y (OPS, 2002), es la violencia contra los animales, que según autoras como López-Ortega (2011) es un predictor de otras violencias.

Los jóvenes ubican las drogas, el alcohol, la falta de vigilancia policial, el abuso de la superioridad física, la discriminación, los contenidos en la televisión, la falta de atención y comunicación entre padres e hijos, los problemas económicos y familiares como las principales causas de la violencia. Respecto a los efectos de la violencia sobre los jóvenes, estos son: emocionales y psicológicos, tales como tristeza, desilusión, miedo, inseguridad, frustración, enojo, traumas, 
odio y pensamientos suicidas; así como relacionales: problemas entre la familia y separaciones, enemistad, resquebrajamiento del sentido de comunidad y el tejido social; y físicos: violaciones, robos, muertes, balaceras y secuestros.

Derivado de estos hallazgos, se denota la necesidad de una estrategia integral de atención a la violencia entre las juventudes que tome en cuenta los retos y necesidades antes descritos y que involucre el mejoramiento de espacios públicos para el deporte y la capacitación, así como la participación ciudadana y talleres especializados en temas de adicciones, violencia intrafamiliar, atención psicológica, igualdad de género, enseñanza de oficios, deportes.

Es necesario además tomar en cuenta el inventario de infraestructura, programas y actores que permitan la coordinación y la eficiencia de los programas públicos ya existentes. Respecto a la infraestructura, existe una desigualdad muy importante en las tres colonias analizadas. Los recursos con los que cuentan los jóvenes para alejarse de la violencia y construir entornos más sanos son mayores en La Independencia, seguidos por La Alianza y, por último, Fomerrey 45, donde hace falta una estrategia de construcción y recuperación de espacios públicos. En el caso de La Independencia, la capacidad de la infraestructura supera su uso, mientras que el caso de La Alianza, la vinculación de programas sociales federales y estatales con el centro comunitario ha atraído mucho a la población y ha intensificado su uso.

En las tres colonias se pudo evidenciar que existen problemas relacionados con las drogas, ya que su acceso es rápido y a precios al alcance de los jóvenes. Destaca la mención del solvente denominado tolueno como droga de uso común entre los jóvenes de estas colonias, lo que coincide con datos del Instituto Nacional de Psiquiatría (Martínez, 2013), que la ubican como una droga alucinógena muy recurrida entre jóvenes de estratos económicos bajos. El tolueno es un solvente químico, cuya inhalación tiene impactos negativos sobre el hígado, los pulmones y causa disfunción cerebral (UNAM, s.f.).

\section{Referencias}

Barreira, C. (2009). Representaciones sobre la violencia entre jóvenes. Estigma, miedo y exclusión. Espacio Abierto, 18(2), 219-234.

Bourdieu, P. (2000). Sobre el poder simbólico. En P. Bourdieu (Ed.), Intelectuales, política y poder (pp. 65-73). Buenos Aires, Argentina: Universidad de Buenos Aires - EUDEBA.

Buvinic, M., Morrison, A. y Orlando, M. (2005). Violencia, crimen y desarrollo social en América Latina y el Caribe. Papeles de Población, 11(43), 167-214.

Cabannes, Y. (2005). Presupuesto participativo y finanzas locales. Presupuestos participativos en ciudades europeas y latinoamericanas. Recuperado de http:// siare.clad.org/fulltext/0049844.pdf

Cámara de Diputados. (1999). Ley del Instituto Mexicano de la Juventud. Última reforma publicada en el Diario Oficial de la Federación, [02.04.2015].

Casas-García, J. M. (2010). Del barrio San Luisito a la colonia Independencia. En C. Contreras (Coord.), Colores y ecos de la colonia Independencia (pp. 17-32). Monterrey, México: COLEF y Municipio de Monterrey. 
Comisión Intersecretarial para la Prevención Social de la Violencia y la Delincuencia (CIPSVD). (2013). Bases del Programa Nacional para la Prevención Social de la Violencia y la Delincuencia e Instalación de la Comisión Intersecretarial. Recuperado de http://www.gobernacion.gob.mx/archivosPortal/pdf/ Bases120213.pdf

Francés, F. y Carrillo, A. (2008). Presupuestos participativos. Guía metodológica de los presupuestos participativos. Recuperado de http://www.redcimas.org/ wordpress/wp-content/uploads/2012/08/t_prepar_guia_metodologica.pdf

Fernández-Areu, I. (2010). Los nahuas de Hidalgo. En C. Contreras (Coord.), Colores y ecos de la colonia Independencia (pp. 71-76). Monterrey, México: COLEF y Municipio de Monterrey.

Galtung, J. (1998). Tras la violencia, 3R: reconstrucción, reconciliación, resolución. Afrontando los efectos visibles e invisibles de la guerra y la violencia. Bilbao, España: Bakeaz/Gernika Gogoratuz.

Galtung, J. y Fischer, D. (2013). Johan Galtung Pioneer of Peace Research. Springer Briefs on Pioneers in Science and Practice. New York, US: Springer. [DOI: 10.1007/978-3-642-32481-9].

Gobierno del Estado de Nuevo León. (2013a). Monterrey: Zonas de Atención Prioritaria Urbana (ZAPU) 2013, por colonia. Indicadores sociodemográficos seleccionados, 2010. Recuperado de http://www.nl.gob.mx/pics/pages/ sdsocial_convocatoriaosc2013_base/zapu.pdf

Gobierno del Estado de Nuevo León. (2013b). Polígonos de las colonias Fomerrey 45, La Alianza y La Independencia [Inédito].

Hernández-de-Frutos, T., Sarabia, B. y Casares, E. (2002). Incidencia de variables contextuales discretas en la violencia 'bullying' en el recinto escolar. Psicothema, 14(Supl.), 50-62.

Instituto para la Seguridad y la Democracia A.C.(INSYDE). (2014). Una aproximación a los costos de la violencia y la inseguridad en México. Recuperado de http:// insyde.org.mx/wp-content/uploads/2014/03/Una_aproximaci\%C3\%B3n_a_ los_costos_de_la_violencia_Insyde_Mexico.pdf

Instituto Nacional de Estadística y Geografía (INEGI). (2016a). Registros administrativos sobre Mortalidad [consulta interactiva de datos]. Recuperado de http:// www.inegi.org.mx/est/lista_cubos/consulta.aspx? $\mathrm{p}=\mathrm{adm} \& \mathrm{c}=4$

Instituto Nacional de Estadística y Geografía (INEGI). (2016b). Estadísticas judiciales en materia penal. Delincuentes sentenciados. Año de registro 2008. [Consulta interactiva de datos]. Recuperado de http://www.inegi.org.mx/lib/olap/ consulta/general_ver4/MDXQueryDatos.asp?proy=esop_delincuentes

Jiménez-Bautista, F. (2012). Conocer para comprender la violencia: origen, causas y realidad convergencia. Revista de Ciencias Sociales, 19(58), 13-52.

Krichesky, M. y Flor, N. (2007). Diagnósticos Participativos Locales. Una estrategia para la defensa por el derecho a la educación. Coordinación General del Material Programa Promoción de Nuevas Comunidades de Aprendizaje. Nueva York, Estados Unidos: Fundación SES y Organizaciones Sociales de la Argentina y del Uruguay.

Korstanje, F. (2009). Planeación participativa: herramientas para el desarrollo local en comunidades rurales. Estudios Agrarios, (41), 9-37. 
López-Ortega, M. (2011). Crueldad hacia los animales: importante indicador de posible crueldad posterior hacia los seres humanos. Revista Psicología.com, 15, 15-37.

Martínez, S. (25 de agosto de 2013). Avanza entre jóvenes el consumo del tolueno, droga letal y barata. Periódico La Jornada. Recuperado de http://www.jornada. unam.mx/2013/08/25/sociedad/

Ministerio de Salud de Argentina. (2004). Municipios y comunidades saludables. Herramientas metodológicas. Recuperado de http://www.munitel.cl/eventos/ seminarios/html/documentos/2011/SEMINARIO_PLAN_COMUNAL_DE_ SALUD_SAN_FELIPE/PPT14.pdf

Nirenberg, O. (2006). El diagnóstico participativo local en intervenciones sociales. Buenos Aires, Argentina: Centro de Apoyo al Desarrollo Local.

Organización de Naciones Unidas (ONU). (2011). Guía para la prevención en barrios. Hacia política de cohesión social y seguridad ciudadana. Santiago, Chile: ONU-HÁBITAT y Universidad Alberto Hurtado.

Organización Mundial de la Salud (OMS). (1986). La salud de los jóvenes: un desafío para la sociedad. Ginebra, Suiza: Organización Mundial de la Salud.

Organización Panamericana de la Salud (OPS). (2002). Informe mundial sobre violencia y la salud: resumen. Washington D.C., US: OPS.

Sanchez-Guerrero, G. N. (2003). Reunión de Planeación Participativa. En Técnicas participativas para la planeación Procesos breves de intervención (pp. 225-252). México D.F., México: Fundacion ICA. A.C.

Scandroglio, B., Martínez, J. M., Martín, M. J., López, J. S., Martín, A., San José, M. C. y Martín, J. M. (2002). Violencia grupal juvenil: una revisión crítica. Psicothema, 14(Supl.), 6-15.

Secretaría de Gobernación (Segob). (2013). Guía de proyectos de prevención social del delito con participación ciudadana. Recuperado de http:// secretariadoejecutivo.gob.mx/work/models/SecretariadoEjecutivo/ Resource/1342/1/images/BProgramadeprevencion_2013.pdf

Universidad Nacional Autónoma de México (UNAM). (s.f.). Hoja de seguridad XVII del tolueno. Recuperado de http://www.quimica.unam.mx/IMG/pdf/17tolueno.pdf 